\title{
Psicología de la instrucción, razonamiento y conocimientos específicos
}

\author{
Mario Carretero, Asunción lópez Manjón, Juan ignacio \\ Pozo, José A. León, Puy Pérez Echeverría, Mikel Asensio \\ Universidad Autónoma de Madrid
}

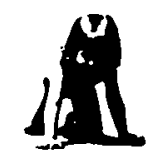

\section{Resumen}

El objetivo de este artículo es presentar la línea de investigación sobre Psicología de la Instrucción desarrollada en la Universidad Autónoma de Madrid. 'Esta línea se ba originado, en términos generales, a partir de los problemas que tenía planteados la teoría de Piaget en tomo al estudio de las operaciones formales. El intento de explicar los resultados anteriores llevaron a considerar fundamental el estudio del contenido en las tareas con estructura lógica y en el razonamiento en general. Los trabajos dentro de esta línea se han visto influidos por los estudios realizados sobre expertos y novatos en áreas de conocimiento específicas y los realizados sobre concepciones previas o erróneas (misconceptions). Concretamente se presentan dos investigaciones especificas. Una sobre las ideas de los alumnos adolescentes en Quimica y otra sobre la comprensión de textos y las variables que influyen en él. En resumen, puede considerarse que bemos pasado de unas preocupaciones más centradas en los procesos cognitivos de razonamiento a otras que tienen más en cuenta la importancia del conocimiento especifico y por tanto de las variables instruccionales.

Palabras clave: Psicología de la instrucción, Razonamiento, Concepciones altemativas en Química, Comprensión de textos.

\section{Psychology of instruction, reasoning and specific knowledge}

\section{Abstract}

The aim of this paper is to introduce a very productive line of research on Psychology of Instruction developed at the Autonoma University of Madrid. The origin of this research work can be found in the problems posed by Piaget's theory around the stage of formal operations. The attempt to explain these results lead us to consider fundamental to study the content of tasks with a logical structure and of reasoning in general. This approach to research bas been influenced by studies on experts and novices' performance in specific areas of knowledge and studies on people's misconceptions. Specifically, two research studies are included. First, a study on adolescent students' ideas about Chemistry, and a second study on text comprebension and variables influencing it. Overall, it seems that our concems have chänged over time. We have moved from cognitive processes in reasoning to other aspects which take into account the importance of specific knowledge and therefore instructional variables.

Key words: Instruction, Reasoning Altemative conceptions Chemistry, Text comprehension. 


\section{INTRODUCCION}

En este artículo vamos a presentar una visión general de los presupuestos fundamentales en los que se basa o se ha basado nuestra línea de investigación sobre Psicología de la Instrucción en la Universidad Autónoma de Madrid llevada a cabo en los diez últimos años, así como algunos desarrollos recientes de dicha línea de trabajo, concretamente los relacionados con la comprensión de la Química y el procesamiento de textos. Un propósito de esta naturaleza requería mucha más. extensión que la que resulta conveniente en un artículo de este tipo. Por tanto, nuestra exposición ha de ser por fuerza resumida y selectiva. En cualquier caso, hemos intentado que sea coherente para que el lector pueda formarse una idea cabal no de todas las cuestiones que nuestro equipo estudia en la actualidad sino más bien de se enfocan'. Por tanto, abordaremos en primer lugar y en términos generales los supuestos básicos que han ido inspirando nuestro trabajo en estos diez años y posteriormente se presentarán brevemente tres investigaciones concretas cuya elaboración específica recae en las diferentes personas indicadas a pie de página. No nos ha parecido útil incluir el contenido de trabajos ya publicados ni otras investigaciones en curso.

Cuando nuestro equipo inicia su andura, aproximadamente en 1982, bajo la direción de Mario Carretero, las cuestiones fundamentales que nos preocupaban procedían de los problemas que tenía planteados la teoría de Piaget en torno al estadio de las operaciones formales (Carretero, 1985; Pozo y Carretero, 1986; Carretero y León, 1991; León y Carretero, en prensa y León, López Manjón y Carretero, 1990). Dichos problemas no sólo eran de naturaleza psicológica sino también educativa. Es decir, no afectaban exclusivamente a si los adolescentes eran capaces de razonar formalmente y resolver problemas lógicos y abstractos de cierta complejidad sino también a la manera de concebir las aplicaciones de la teoría piagetiana a la educación. Aunque estos dos problemas se encuentran muy relacionados conviene tratarlos por se- parado. En lo que se refiere a las cuestiones puramente psicológicas, a comienzos de los ochenta se había producido suficiente investigación empírica como para saber que los distintos problemas utilizados por Inhelder y Piaget (1955) resultaban de muy distinta dificultad para los adolescentes e incluso para los adultos. En definitiva, resultaba muy difícil mantener la noción de estadio tal y como se sostenía en el caso de estadios anteriores del desarrollo, por ejemplo, en el correspondiente a las operaciones concretas. Curiosamente una fuente de críticas a este respecto vino de la psicología cognitiva y más concretamente de los estudios sobre razonamiento de Wason y Johnson-Lair. Estos psicólogos británicos mostraron que algunas tareas lógicas aparentemente sencillas y de naturaleza hipotético-deductiva planteaban enormes dificultades incluso a adultos con formación universitaria. La razón fundamental era que los sujetos no comprendían en absoluto la utilidad de la falsación como estrategia de razonamiento y por el contrario tendían con mucha mayor frecuencia a utilizar la estrategia de verificación. En definitiva, la crítica a la que se enfrentaba el pensamiento formal ya no era más o menos puntual sino que llegaba a cuestionar la imagen del adolescente y el adulto como un aprendiz eficaz y competente de científico y metodólogo. Con el tiempo, esta sombra de duda acerca de la racionalidad se verá acrecentada con los trabajos posteriores de autores como Tversky y Kahneman (Carretero y García Madruga, 1984; García Madruga y Carretero, 1986; Carretero, Pérez Echeverría y Pozo, 1986).

En cualquier caso, para el asunto que ahora nos ocupa conviene insistir en que nuestra preocupación fundamental no eran las diferentes subetapas evolutivas por las que discurría el desarrollo intelectual durante la adolescencia. Tampoco nos preocupó prioritariamente la utilidad de la lógica para formalizar el razonamiento de los alumnos, aunque realizamos algunos trabajos sobre solución de problemas lógicos, tanto en sujetos normales como con minusvalías sensoriales. Nuestra preocupación fundamental residía en dos cuestiones. Por un lado en el estudio de 
la importancia del contenido en el razonamiento y más concretamente en la investigación de contenidos que apenas se habían estudiado en relación con el pensamiento formal como era el caso de las Ciencias Sociales. Por otro lado, en el estudio del razonamiento desde un punto de vista más funcional a como lo había estudiado la Escuela de Ginebra. Es decir, excesivamente ligado a las estructuras lógicas. En este sentido cobraba para nosotros especial importancia la noción de estrategia que había comenzado a utilizarse en algunos desarrollos recientes de la Escuela de Ginebra (véase, por ejemplo, KarmiloffSmith e Inhelder, 1974). A este interés por los aspectos funcionales del razonamiento no eran ajenas las influencias de la filosofía de la ciencia a través de los autores mencionados anteriormente.

El interés por el problema del contenido nos llevó al estudio de la solución de tareas formales con contenido histórico (Pozo y Carretero, 1983). Nuestro objetivo inicial en este ámbito fue analizar en detalle la aplicación de diferentes estrategias de verificación y falsación - relacionadas con lo que Piaget denominaba «control de variables»- a problemas de contenido social. Pudimos comprobar la dificultad específica que este tipo de problemas tenía para los adolescentes, que en un buen número de casos les parecían incluso más complicados que las tareas relacionadas con las distintas Ciencias Naturales. Peto quizá las consecuencias más importantes para el curso de nuestras investigaciones fueron las siguientes: a) los problemas para una adecuada comprensión de las Ciencias Sociales no dependían sólo del razonamiento formal, es decir, de estrategias generales de razonamiento, sino que también existían numerosas deficiencias en la asimilación de aspectos conceptuales básicos, que para nuestra sorpresa no habían sido apenas estudiados (iy siguen sin estarlo suficientemente hasta la fecha!) como es el caso del tiempo o la casualidad histórica, y b) si en algunos aspectos el contenido histórico planteaba dificultades específicas, ¿qué papel tenían entonces las habilidades generales del pensamiento formal? El última instancia, tal y como solía plantearse en aquel momento, el pensamiento formal parecía muy poco formal en la medida en que la forma no era el factor más influyente que determinaba la actuación de los sujetos.

En definitiva, tanto los trabajos sobre la Historia así como algunos otros que referimos más adelante fueron alimentando en nosotros la convicción de que el contenido de los problemas debía ser tratado de una manera muy diferente a como lo había hecho la Escuela de Ginebra, que había dado prioridad a la estructura de las tareas. Por ejemplo, en nuestras investigaciones sobre tiempo y causalidad históricas (Carretero, Pozo y Asensio, 1983; Asensio, Carretero y Pozo, 1989; Carretero, Asensio y Pozo, 1991) encontramos que ni las estructuras ni las estrategias de razonamiento eran demasiado útiles para dar cuenta de la representación que tienen los alumnos de dichas cuestiones. Por otro lado, cuanto más tratábamos estas cuestiones, no sólo en investigaciones sino en numerosos cursos para profesores, más nos convencíamos de que para entender adecuadamente los procesos de comprensión de conocimientos específicos - sean de Historia, de Física o de cualquier materia - resultaba insuficiente una aproximación psicológica y era totalmente imprescindible una comprensión disciplinar de la materia en cuestión. De dicha comprensión disciplinar podríamos esperar un análisis en el que basar adecuadamente los problemas derivados del contenido de las tareas.

Anteriormente hemos mencionado de pasada la aplicabilidad de la teoría de Piaget a la educación, puesto que nuestra línea de investigación nace vinculada a dicha orientación. Como es sabido, a finales de los setenta surgen importantes críticas a lo que podía considerarse una visión un tanto simplificada de las relaciones entre las investigaciones de la Escuela en Ginebra y el mundo educativo. Dichas críticas mantenían, entre otras cuestiones, que la aportación de tipo evolutivo de Piaget no podía constituir la funda. mentación esencial de la innovación educativa precisamente por su propio carácter evolutivo y escasamente instruccional. En otras 
palabras, la teoría de Piaget nos informa acerca de cómo se produce el desarrollo cognitivo pero apenas nos proporciona conocimientos útiles acerca de cómo se lleva a cabo el aprendizaje en general y más concretamente el escolar. Por supuesto, es bien cierto que el desarrollo cognitivo es muy importante para que se produzca el aprendizaje pero no es el único requisito. En alguna otra ocasión hemos considerado esta cuestión como la confusión implícita en muchas investigaciones entre el niño y el alumno (Carretero, 1989). Desde este punto de vista, la cuestión de los contenidos anteriormente mencionada resultaba de enorme pertinencia. Lo que los alumnos deben aprender en clase son contenidos específicos que aunque tengan una serie de regularidades en común, también plantean muchas diferencias según las distintas materias escolares.

Con esta perspectiva general creemos que puede entenderse con cierta propiedad la utilidad y pertinencia que tuvieron para nosotros -y siguen teniendo en la actualidadlas aportaciones de dos líneas de investigación de gran arraigo en el contexto internacional. Nos referimos a los trabajos sobre expertos y novatos y a las investigaciones sobre concepciones erróneas o previas (misconceptions). En ambos casos hemos hallado un grado de análisis del contenido de los problemas que permite estudiar mejor la representación y solución tanto de los sujetos como de los alumnos. La influencia de dichas orientaciones en la investigación educativa de las últimas décadas es sobradamente conocida pero en cualquier caso quizá convenga comentar que los trabajos sobre misconceptions han sido la espina dorsal de la mayoría de los proyectos renovadores sobre enseñanza de las ciencias y que han mostrado que una persistencia de la concepciones erróneas ante muchos fenómenos científicos es moneda corriente no sólo entre los niños y adolescentes sino entre los adultos. De esta manera su análisis detallado nos permite predecir la representación que puede surgir en un determinado contexto educativo habida cuenta de su generalidad y ubicuidad (Pozo y Carretero, 1987 y Pozo, 1987).
Por su parte, las investigaciones sobre expertos y novatos ha sido una de las bases más sólidas sobre las que se ha desarrollado la psicología de la instrucción en las últimas décadas. Con una influencia muy clara del procesamiento de la información y de la psicología congnitiva en general esta distinción ha permitido determinar que la diferencia entre un experto y un novato en un determinado dominio de conocimiento no sólo es una cuestión cuantitiva sino también cualitativa y que afecta a los procesos cognitivos básicos como son la percepción, atención y memoria a corto y largo plazo. Es decir, un experto en geografía física, pongamos por caso, no sólo ha acumulado mayor cantidad de información que una persona ignorante del tema sino que la tiene organizada con una estructuración mucho más eficiente que permite recuperarla mejor y sobre todo enfrentarse con problemas de este tipo recurriendo a reglas más apropiadas basadas en principios de carácter nomotético. Por otro lado, el experto puede obtener un mejor rendimiento de su memoria a corto plazo puesto que agrupa la información nueva en unidades significativas de mayor densidad informativa.

De esta manera, el enfoque de expertosnovatos supone una reconsideración novedosa de los problemas que tenía planteados no sólo la teoría de Piaget sino cualquier posición sobre el desarrollo cognitivo. Para ver su pertinencia al respecto basta con considerar al niño en desarrollo como un sujeto que va acumulando experiencia y que de hecho en algunos terrenos puede tener más que un adulto, dependiendo del tipo de aprendizaje que realice. Por el contrario piénse en el adulto como un novato que en algunos ámbitos puede poseer tan pocos conocimientos como un niño. Por ejemplo, cuando un adolescente o un adulto no realizaba adecuadamente una tarea formal como la relativa a la oscilación del péndulo, ¿se debía a una incapacidad para aplicar los principios del razonamiento formal o una falta de dominio de los principios de la física? En nuestra opinión ambas cuestiones se encontraban entremezcladas en las investigaciones piagetianas. No parece que haga falta insistir en las implicaciones edu- 
cativas de todo lo que acabamos de exponer. Baste decir que de como sea la respuesta a la pregunta que acabamos de hacer depende que se defienda una aplicación educativa u otra de las investigaciones sobre desarrollo cognitivo. En un caso daremos prioridad a enseñar habilidades de pensamiento y en el otro, a los contenidos. Como quizá pueda imaginarse, nuestra posición al respecto es justamente la intermedia. Es decir, resulta fundamental enseñar a pensar y razonar mejor pero es preciso hacerlo concediendo importancia a los contenidos porque no es posible hablar de razonamiento en abstracto, al menos desde el punto de vista educativo (Pozo, Asensio y Carretero, 1989).

Por otra parte, una de las críticas que ha recibido en los últimos años la investigación sobre misconceptions ha sido el panorama fragmentario que presentan sobre el conocimiento del sujeto. La abundancia de trabajos sobre el tema no ha llevado, sin embargo, a formular modelos consistentes que expresen qué tienen en común dichas concepciones. Nosotros mismos hemos investigado las características de dichas concepciones en dominios tan diferentes como las Ciencias Sociales, la Física (Carretero, 1984; Pozo, 1987, y Pozo y Carretero, 1991) y la Medicina (López Manjón, 1991; López Manjón y Carretero, en prensa). Tanto en el caso de la Física como de las Ciencias Sociales hemos podido comprobar cómo a menudo existe una clara relación entre lás concepciones previas acerca de un mismo dominio. Por otro lado, también hemos mantenido una distinción entre dichas concepciones - en la medida en que actúan como teorías causales- y las estrategias de razonamiento que el sujeto aplica al resolver un problema. Nuestros resultados indican que dichas estrategias resultan una condición necesaria pero no suficiente para enfrentarse con problemas de cierta complejidad en ambos dominios.

En el apartado que sigue puede verse con mayor detalle una investigación specífica sobre las representaciones espontáneas de la Química y sus implicaciones para su enseñanza. En el siguiente apartado se presenta otro trabajo en curso acerca del recuerdo de tex- tos y de las variables que influyen en su comprensión. En este último es evidente la influencia de los trabajos cognitivos al respecto que han sido, y siguen siendo, particularmente florecientes en la última década. Aunque se trata de estudios sobre temáticas diferentes, creemos que constituyen un buen ejemplo de cómo han evolucionado nuestros intereses y objetivos. En términos generales puede decirse que hemos pasado de unas preocupaciones más centradas en los procesos de razonamiento y desarrollo cog. nitivo a otras que tienen más en cuenta la importancia del conocimiento específico y por tanto las variables instruccionales.

\section{LA COMPRENSION Y EL APRENDIZAJE DE LA QUIMICA POR LOS ADOLESCENTES: DE LAS TEORIAS IMPLICITAS A LAS TEORIAS CIENTIFICAS ${ }^{2}$}

La mayoría de las investigaciones realizadas en torno al aprendizaje y la enseñanza de las ciencias durante las últimas décadas se han basado en un supuesto constructivista. Según este supuesto, asumido también por el Diseño Curricular Base del M.E.C., el alumno construye sus conocimientos científicos por medio de sus propios procesos cognitivos y tomando como punto de partida la activación de los conocimientos que tiene ya almacenados o ideas previas. Asumir este principio lleva consigo reconocer la necesidad de identificar e investigar la naturaleza y el contenido de las ideas sobre las ciencias que mantienen los alumnos.

Aunque, actualmente, existe un consenso en torno al supuesto "constructivista», este concenso parece desaparecer cuando intentamos precisar un poco más su significado. Parafraseando la célebre fràse de Ausubel, Novak y Hanesian (1978), podríamos decir que hay diversas formas de «averiguar» $\mathrm{y}$ conceptualizar «lo que el alumno ya sabe», cada una de las cuales tiene repercusiones muy diferentes cuando se concreta en opciones curriculares determinadas. 
Los conocimientos previos de los alumnos, ¿estadios o ideas aisladas?

De forma general se podría decir que las investigaciones sobre los conocimientos de los alumnos acerca de la ciencia se han basado fundamentalmente en dos opciones teóricas alternativas. Por un lado, una buena parte de los trabajos ha partido de la teoría piagetiana de las operaciones formales (Inhelder y Piaget, 1955; Shayer y Adey, 1981) según la cual la comprensión del alumno de los contenidos específicos estaría determinada por su nivel de desarrollo cognitivo. Por otro lado, otras investigaciones se han basado en el enfoque más reciente de las ideas previas o concepciones alternativas de los alumnos sobre los fenómenos científicos (por ejemplo, Archendhold et al., 1980; Driver, Guesne y Tiberghien, 1985; Hierrezuelo y Moreno, 1988), que parte del estudio aislado de diferentes ideas en dominios distintos, sin establecer apenas conexiones o vínculos entre la construcción de distintos conceptos ni siquiera dentro de la misma área del currículo.

Aunque los dos enfoques coinciden en su visión constructivista del aprendizaje, difieren entre sí en lo referente a otras presuposiciones que, a su vez, entrañan opciones curriculares claramente distintas (véase Pozo y Carretero, 1987; Pozo et al., 1991a). La teoría de las operaciones formales de Piaget (Inhelder y Piaget, 1955) partía de que en la adolescencia, los alumnos construyen el mundo basándose en una mente altamente organizada, estructurada de un modo homogéneo de acuerdo con ciertas capacidades lógicas subyacentes. La comprensión de la ciencia dependería, según Piaget, del nivel de desarrollo cognitivo general alcanzado. La teoría de Piaget proporciona la imagen de un alumno con una mente homogénea y organizada que permite realizar predicciones sobre su futuro rendimiento en diferentes áreas. Aunque estas predicciones no siempre se cumplan, Piaget ha legado a la psicología un buen número de «regularidades». en la conducta de los alumnos que cualquier teoría debe explicar (Case, en prensa; Flavell, 1982).

No obstante, hay muchos otros trabajos que ponen en duda la propia existencia de estadios en el desarrollo cognitivo y más concretamente la existencia del estadio operacional formal (véase Cárretero, 1985). Los datos indicativos de que un escaso número de sujetos adolescentes e incluso adultos resuelven formalmente tareas científicas, unidos a los que muestran la inconsistencia en el uso del pensamiento formal por un mismo sujeto en diferentes contextos han hecho que el interés de los investigadores $-\mathrm{y}$ de los educadores - se desvíe hacia los conocimientos específicos que afectan al rendimiento del alumno en una tarea determinada.

En la investigación sobre enseñanza de la ciencias este interés creciente por los conocimientos específicos ha venido de la mano del segundo de los grandes enfoques antes mencionados: el de las ideas previas. Los trabajos surgidos dentro de este enfoque han estudiado cómo entienden los alumnos nociones científicas específicas. Las concepciones de los alumnos sobre la ciencia se estudian hoy como ideas aisladas, inconexas, cuyo único nexo - y de referencia teórica- son las disciplinas científicas con respecto a las cuales son «alternativas». Si de constructivismo y de construir estamos tratando, es difícil construir un modelo de la mente del alumno con elementos tan dispersos. El enfoque de las concepciones alternativas ha supuesto una cierta «desintegración» del alumno, que ha pasado de poseer un sistema cognitivo organizado y predecible a disponer de un número no determinado de concepciones poco conectadas entre sí y por tanto difícilmente predecibles.

Como veíamos antes, esta tendencia se justifica en parte por los datos que ponen en duda la existencia de estructuras cognitivas homogéneas en el pensamiento de los alumnos (véase Carretero, 1985). Sin embargo, el conocimiento científico de los alumnos tampoco parece ser tan heterogéneo o dispar como el enfoque de las concepciones alternativas supone actualmente. Aunque no se han hallado correlaciones entre tareas que según Piaget debían estar altamente relacionadas, se han observado correlaciones estadísticamente significativas entre tareas formales que des- 
de el punto de vista de las concepciones alternativas no serían predecibles (por ejemplo, Demetriou, Efklides y Gustaffson, en prensa; Lawson, 1977; Shayer y Adey, 1981). Además, los pocos trabajos que han intentado establecer correlaciones entre las concepciones alternativas de los alumnos en diversas tareas han observado que éstas no son completamente independientes, pero tampoco constituyen un sistema de conjunto único y general, ya que tienen un nivel de coherencia variable (por ejemplo, Engel Clough y Driver, 1986; Jiménez Aleixandre, 1990, y Pozo, 1987).

\section{Un nivel de análisis intermedio: las estruc- turas conceptuales o teorías implícitas}

En general, los datos que hemos descrito brevemente parecen reclamar niveles de generalidad y homogeneidad intermedios entre los previstos por Piaget y las concepciones alternativas (Case, en prensa). Según este análisis, el pensamiento científico no constituiría un sistema tan homogéneo como la teoría piagetiana predecía pero tampoco tan heterogéneo como algunos otros autores suponen. No obstante, este problema no es nuevo ni específico de la comprensión de la ciencia. Parece estar muy ligado al problema de los estadios en psicología evolutiva (Flavell, 1982; también Carey, 1985) y parece requerir teorías o modelos que expliquen tanto la generalidad como la especificidad del conocimiento (Bidell y Fischer, en prensa). La aparición de las teorías neopiagetianas constituye un intento de explicar esas regularidades recurriendo a ciertos rasgos estructurales o funcionales que permitirían predecir su actuación en diversas tareas.

Otra forma de explicar las regularidades en la comprensión de la ciencia por parte de los alumnos es recurrir a estructuras conceptuales de un nivel de generalidad intermedio entre las estructuras lógicas piagetianas y las dispersas concepciones alternativas. Los intentos de Carey (1985), o más recientemente de Case (en prensa), así como la referencia a teorias implicitas de los alumnos (por ejemplo,
Pozo, 1987; Rodrigo, 1985) son intentos de este tipo.

El enfoque de las «teorías implícitas» se ha basado en recientes conceptualizaciones de la psicología cognitiva sobre la forma de representar el conocimiento y el mundo que nos rodea. Distintos trabajos parecen mostrar que los estudiantes poseen una serie de teorías constituidas por distintos conceptos y nexos que establecen relaciones entre ellos. Como las teorías científicas, estas teorías «personales» tienen como objetivo la interpretación y predicción del mundo circundante (véase, por ejemplo, Furham, 1988). No obstante, las teorías científicas y las teorías personales sobre la ciencia difieren entre sí en muchos otros aspectos (una exposición detallada puede encontrarse en Pozo, Pérez Echeverría, Sanz y Limón, 1992). Entre ellos cabe destacar el hecho de que las representaciones de los sujetos expertos y novatos no sólo se diferencian por su contenido sino también por su organización. En este sentido, las teorías personales se caracterizan tanto por poseer rasgos similares a las «categorías naturales» (véase Rosch, 1978; Scholnick, 1983) y ser más inestables que las teorías científicas como por tener un carácter implícito similar al atribuido a los «modelos mentales» (por ejemplo, Holland, Holyoak, Nisbett y Thagard, 1986; Johnson-Laird, 1983). Expresado con otras palabras, las teorías personales sobre la ciencia suelen estar constituidas por conceptos vagos y difusos que subyacen a la acción. No son conocimientos explícitos que surjan tras una reflexión y se puedan comunicar fácilmente de forma verbal.

Por otro lado, las diversas teorías implícitas mantenidas por los alumnos presentan una serie de rasgos estructurales comunes, que podrían actuar como obstáculos epistomológicos que deberían ser superados para que los alumnos comprendieran las teorías científicas. Estos rasgos o restricciones estructurales serían opuestos a algunos de los esquemas operatorios formales descritos por Piaget (Inhelder y Piaget, 1955). Según el propio Piaget, estos esquemas tendrían un rango de generalidad intermedio entre las características formales generales y las nociones científicas 
específicas. Un análisis del contenido de las teorías implícitas de los alumnos en diversos dominios de la ciencia muestra que en muchos casos se caracterizan por la dificultad de superar algunos problemas conceptuales que requerirían el uso de determinados esquemas operatorios formales, similares a los propuestos por Piaget. En este sentido puede tener cierto valor heurístico estudiar si en el paso de sus teorías personales implícitas a las teorías científicas, los alumnos deben aprender a utilizar esquemas generales en el análisis de las relaciones causales, sin los cuales la teoría científica no podría ser correctamente comprendida (Pozo et al., en prensa). Así resulta útil analizar las ideas de los alumnos en una disciplina concreta -en nuestro caso la Química- partiendo de ese nivel de generalidad intermedio, próximo de alguna forma al concepto piagetiano de «esquema operatorio formal» (Pozo, 1988; Pozo et al., 1992).

\section{Tres núcleos conceptuales en la comprensión de la química}

A partir de una revisión y análisis detallado de las investigaciones realizadas hasta la fecha (Pozo, Gómez Crespo, Limón y Sanz, 1991a), hemos identificado en el área de la químia tres nociones o estructuras conceptuales generales, que estarían emparentadas tanto con los esquemas operatorios formales de Inhelder y Piaget (1955), como con las concepciones específicas de los alumnos sobre la química. Estos tres núcleos, vinculados directamente con gran parte de las dificultades y «errores conceptuales» que aparecen en el estudio de esta área, serían respectivamente la comprensión de la materia como algo discontínuo, la conservación de propiedades no observables y la cuantificación de relaciones. Formarían un tronco común del que se derivarían otras muchas nociones más específicas, permitiendo establecer una jerarquía en las dificultades de comprensión de esta área de la Ciencia por parte de los alumnos.

Desde el punto de vista disciplinar o epistemológico, estos tres núcleos o estructuras conceptuales son de gran importancia para la construcción de los conceptos y leyes que conforman la química. La primera de ellas, la noción de discontinuidad, es fundamental para comprender cómo está formada la materia y sus propiedades. La materia, tal como lo describe la ciencia, está formada por partículas que pueden moverse, unirse o combinarse entre sí, no existiendo absolutamente nada entre ellas, lo que implica la idea de vacío. Sin embargo, son numerosos los ejemplos que muestran que los alumnos adolescentes y los adultos no entrenados tienden a mantener de modo bastante generalizado y tenaz teorías implícitas según las cuales la estructura no observable de la materia tiene propiedades similares en lo esencial a sus características ob. servables (Brooks et al., 1983; Driver, 1985; Llorens, 1991), lo cual, a su vez, les lleva a pensar que una de las características de la materia es la continuidad.

Una de las posibles causas de esta perseverancia es que este tipo de creencias es coherente con las características del pensamiento causal de los estudiantes (Pozo, Sanz, Gómez Crespo y Limón, 1991b). Los alumnos conciben la materia tal como la perciben. Aunque la dependencia de los sentidos disminuya con el desarrollo cognitivo, es aún bastante fuerte para dificultar la comprensión de un mundo compuesto por unidades invisibles y discretas, en clara oposición a la realidad percibida. Además, aunque los alumnos lleguen a vislumbrar en algunas tareas o situaciones la posibilidad de un mundo discontinuo tienden a regresar a sus ideas intuitivas, por dos razones. Una primera razón es la creencia en la semejanza entre las causas y los efectos. Sí, la «conducta» de la materia depende de su estructura íntima, nada más «razonable» que atribuir a esas causas no observables (partículas) propiedades similares a las que poseen sus efectos (mundo observable). Pero hay un segundo factor, en nuestra opinión mucho más importante, que no ha recibido excesiva atención a la investigación realizada hasta la fecha. Pensamos que tras es. tas dificultades subyace un problema de representación de lo no observable. En la medida en que el alumno debe abandonar los indicios preceptivos como fuente de represen- 
taciones con respecto a la estructura de la materia, carece de ningún otro código de representación alternativo. Dicho en otras palabras, si las imágenes que los alumnos perciben del mundo no son suficientes para comprender la estructura de la materia, la enseñanza no logra proporcionarles sistemas de representación alternativos que les permitan comprender su naturaleza.

La segunda estructura o núcleo conceptual al que aludíamos al comienzo de este apartado, la conservación, es necesaria para comprender las transformaciones de la materia, los cambios físicos y los cambios químicos. A diferencia de los cambios físicos que experimenta la materia, en los cambios quími$\cos$, la identidad de las sustancias que participan en el proceso se modifica, cambiando su estructura microscópica. En estos procesos se conserva el número total de átomos de cada elemento presentes al principio y al final. Además, los cambios químicos no son reversibles por medios físicos. También en este caso existen numerosos ejemplos que muestran que las interpretaciones dadas por los alumnos están claramente basadas en aquellos hechos que son capaces de percibir (Driver, Guesne y Tiberghien, 1985; Furió y Hernández, 1983; Seré, 1985, 1986). De acuerdo con el pensamiento causal del alumno hay un predominio de todo aquello que es observable sobre lo no observable.

Como los estudios piagetianos han mostrado con claridad, casi la totalidad de las constantes y conservaciones que podemos establecer con respecto al mundo que nos rodea son el producto de nuestro esfuerzo cognitivo por comprender el mundo y, por tanto, lejos de ser una intuición, son una construcción. Nuestro pensamiento tiende a centrarse más en lo que cambia que en lo que permanece. Por ello debemos ir comprendiendo que tras los cambios aparentes hay algo que permanece. En aquellos casos en los que sólo el cambio es perceptible, pero no lo que se conserva, Piaget consideraba que se requerían las operaciones formales para comprender esa noción. Sea o no así, lo que se conserva tras un cambio químico, sea una reacción o sea una disolución, pertenece al mundo de lo no observable, nos remite una vez más a esas minúsculas partículas que componen la estructura oculta de la realidad. Por consiguiente, este problema es uno de los más difíciles de superar en la comprensión de la química, impide en último extremo comprender la propia noción de cambio químico y, en definitiva, la propia estructura química de la realidad.

Por último, al hablar del tercer núcleo, la cuantificación de relaciones, queremos referirnos a la representación cuantitativa de las leyes físico-químicas y a su aplicación práctica. La aplicación cuantitativa de las leyes químicas constituye una de las partes más importantes de esta ciencia, probablemente la que más dificultades presenta para los estudiantes. La gran mayoría de los cálculos utilizados para aplicar las leyes químicas elementales que se utilizan en la enseñanza secundaria se pueden realizar aplicando relaciones de proporcionalidad.

La proporción es un esquema ampliamente descrito por Inhelder y Piaget (1955) y supone el conocimiento de la relación de igualdad entre dos razones y de la existencia de una relación multiplicativa entre sus miembros. Según Inhelder y Piaget la comprensión de las proporciones depende totalmente de la construcción de las operaciones formales. Antes de alcanzar este tipo de razonamiento, los niños utilizarían estrategias de cálculo menos elaboradas que abarcan desde la estrategia cualitativa, pasando por la estrategia aditiva y acabando con la estrategia por correspondencia o por construcción.

No obstante, el cálculo de proporciones plantea grandes dificultades a los estudiantes adolescentes cuando se aplica a la resolución de problemas químicos, sobre todo, teniendo en cuenta el número de proporciones diferentes y sucesivas que aparecen en éstos. Numerosas investigaciones han puesto de manifiesto la relación existente entre el rendimiento en Química y el manejo de cálculos proporcionales y son también numerosos los ejemplos de las dificultades que encuentran nuestros alumnos en su aplicación (véase, por ejemplo, Anamuah-Mensah, 1986; Frazer y Servant, 1987: Serrano y Blanco, 1988). 
No obstante estas dificultades no parecen ser exclusivas del campo de la química. Distintos trabajos muestran que los sujetos adolescentes y adultos utilizan diversas estrategias de cómputo para solucionar problemas de tipo correlacional (véase para una revisión Pérez Echeverría, 1990; Sanz, 1991). Un mismo sujeto varía en el tipo de estrategia usado dependiendo de la tarea (véase, por ejemplo, Carretero, Pérez Echeverría y Pozo, 1986; Sanz, 1991). Por tanto, parece que el cálculo de proporciones no es un problema de competencia, es decir, de que los alumnos no sepan utilizarla en absoluto sino un problema de actuación que depende de factores que afectan tanto a la tarea como al sujeto (para una revisión de estos factores véase Sanz, 1991; Tourniaire y Pulos, 1985).

\section{UN MODELO DE INTERVENCIÓN EN LA MEJORA COMPRENSIVA DE UN TEXTO $^{3}$}

La adopción de un enfoque cognitivo de la lectura y su comprensión ha permitido, en estos últimos años, un avance espectacular, tanto en su profundización teórica como en su aplicación práctica. En efecto, sobre la base de un enfoque constructivista de la memoria y de la elaboración de herramientas analísticas con las que inspeccionar y diseccionar la estructura del material escrito, se diseñaron en los años setenta diversos modelos de análisis estructurales de la prosa, de los que sobresalen los análisis proposicionales propuestos para el estudio de textos narrativos y expositivos (Kintsch, 1974; Meyer, 1975; Kintsch y van Dijk, 1978) y los que se centraron en el estudio de historias populares y que se denominaron «gramáticas de las historias» (Rumelhart, 1975; Mandler y Johnson, 1977; Thorndyke, 1977; Stein y Glenn, 1979). Asimismo, la introducción de conceptos y constructos como la teoría del esquema o los modelos mentales, están permitiendo explicar de manera coherente cómo se representa y comprende el conocimiento en la mente del sujeto, cómo se almacena, o qué procesos y estrategias participan en la comprensión y retención del material escrito. Una buena prueba de ello son los modelos explicativos e interactivos propuestos por algunos autores durante la década de los ochenta y que han considerado, además de la estructura del texto, los aspectos constructivos del lector (John. son Laird, 1983; van Dijk y Kintsch, 1983; Meyer, 1984, 1985a y b; Garnham, 1987; Kintsch, 1988).

Esta tendencia dominante de los modelos interactivos de la actividad lectora ha impreg. nado, en estos últimos años, tanto en trabajos que se han interesado por el análisis de las estrategias utilizadas por los lectores como por su enseñanza, esto es, en la elaboración de modelos de instrucción encaminados a mejorar su aplicación. Actualmente se considera que las dificultades para comprender lo leído se deben, al menos en parte, a que las estrategias de comprensión y de prendizaje que poseen los sujetos son inapropiadas o inexistentes para realizar correctamente la tarea. Puede deberse incluso a que el lector no sea consciente de las demandas de la tarea. Las implicaciones en este cambio de orientación están cuestionando también el modo de enseñar. El enseñante, además de transmitir al alumno los contenidos propios de la asignatura que imparte, debe ser capaz de incitar al alumno a que trabaje con esos contenidos de manera activa, implicándole directamente en la tarea.

Si un objetivo prioritario de este tipo de investigaciones es tratar de mejorar la comprensión de estos sujetos, se hace necesario conocer de antemano la capacidad que los sujetos poseen en la adquisición y uso de la información y cuestionarse si esta capacidad es susceptible de mejorarse con el entrenamiento de estrategias que permitan procesar la información de una manera más eficaz.

Un cuerpo de investigaciones se ha centrado en analizar las diferentes estrategias que los lectores competentes y no tan competentes desarrollan ante la lectura y comprensión de un texto. Las conclusiones de estos trabajos sugieren que los buenos lectores se muestran más activos que los lectores con pobre nivel de comprensión cuando procesan la in- 
formación del texto. Estas diferencias se producen frecuentemente, porque, al contrario que los lectores más consumados, los menos competentes no son capaces de organizar correctamente la información nueva, lo que les imposibilita relacionarla con la que ya se posee. En muchos casos estas diferencias pueden estar motivadas por la ineficacia o inhabilidad que muestran estos lectores a la hora de seleccionar e implementar las estrategias adecuadas en la comprensión del material escrito. En general, todos coinciden en alguna medida con las características globales de la representación que obtienen los diferentes sujetos en su memoria tras la lectura de un texto. Frente a la representación mental de los lectores consumados, caracterizada por una representación coherente que distingue diferentes niveles de importancia en el contenido del texto, reteniendo las ideas que determinan la macroestructura del mismo, la de los lectores menos competentes, por el contrario, se presenta bajo la forma tema más detalles (Scardamalia y Bereiter, 1984). Esta última se caracteriza por una representación mental del tema general del texto acompañada por una colección inarticulada de detalles.

Así desde esta perspectiva, se han identificado estrategias propias de los lectores competentes y que han sido denominadas de diferentes formas, estrategias organizativas (Just y Carpenter, 1987), de comprensión (Spring, 1985), estrategia significativa (Loman y Mayer, 1983), codificación asimilativa (Mayer, 1985) o estrategia estructural (Meyer, 1984). Estas estrategias están encaminadas a generar o activar los esquemas que posee el sujeto, requieren en el lector la existencia de un conocimiento previo adecuado y de la disponibilidad de un uso estratégico de esos conocimientos. Dentro de ellas se incluye aquella destreza que permite al lector relacionar la nueva información leída con la que ya posee o aquella habilidad para identificar las principales relaciones lógicas del pasaje, que permitan conectar las diferentes proposiciones del texto dentro de una secuencia coherente. Esta noción sobre la estructura del texto permite al lector consumado la construcción de una representación o modelo mental de la informa- ción leída más coherente y organizada (Mayer, 1985). De esta forma, el lector comprende el pasaje sin necesidad de recordar la información específica.

Por el contrario, el lector inmaduro presenta otros tipos de estrategias, como la estrategia mecánica (Loman y Mayer, 1983), estrategia en lista por defecto (Meyer, 1984) o ante tareas de resumen, la estrategia suprimir y copiar propuesta por Brown, Day y Jones (1983). Estas estrategias se caracterizan por una representación fragmentada y lineal y se utilizan cuando el lector concibe el pasaje como una lista de hechos o acontecimientos separados que han de ser memorizados. La estrategia en lista por defecto, por ejemplo, describe la situación de un lector que, aun pretendiendo extraer la misma representación que la propuesta por el autor, carece de un plan estratégico de procesamiento. En este caso, el lector parece operar como si la meta de la lectura fuese recordar «algo» del texto, lo que podría comprobarse en el protocolo de recuerdo como una relativa insensibilidad a la organización jerárquica del contenido textual. En consecuencia, el lector menos competente concibe el texto como una lista de elementos aislados, careciendo de toda organización lógica (Meyer, 1984).

La presencia de estos tipos de estrategias han sido puestas de manifiesto en trabajos recientes dirigidos a analizar diferencias entre lectores «expertos» $y$ «novicios» (León, 1989; León y Carretero, en prensa). Los resultados obtenidos en estos estudios indican que las diferencias esenciales entre las respuestas emitidas por los adultos expertos y alumnos de $1 .^{\circ}$ de BUP aparecen en la calidad de las respuestas, siendo las del experto más selectivas respecto a la macroestructura del texto y mejor organizadas desde el punto de vista de la estructura original del pasaje. Este conocimiento implícito sobre la estructura del texto se revela como uno de los factores claves responsables de esta superioridad. El lector maduro parece utilizar hábilmente este conocimiento tanto en la representación de la nueva información como en la recuperación de esa información a la hora de elaborar sus respuestas. Al mismo tiempo, el uso de este ti- 
po de conocimiento revierte en una mayor estabilidad del recuerdo con el paso del tiempo.

Estas diferencias nos sugieren a la hora de planificar un modelo de instrucción que incida en la mejora de la comprensión del material escrito en lectores menos competentes, basarnos en la asunción de que estos lectores pueden beneficiarse de la enseñanza de aquellas estrategias que los lectores maduros utilizan de forma natural (Oakhil y Garnham, 1988). Más específicamente, si se interviene en procedimientos específicos que induzcan al lector un conocimiento sobre la estructura textual y su utilización, así como la implantación de sistemas de control de las propias actividades que pretenden promoverse, pueden mejorar el comportamiento lector en aquellos sujetos que no poseen estrategias de comprensión o si las poseen, no las utilizan adecuadamente. En este sentido, podemos presumir que el comportamiento del lector menos capaz se aproximaría en tal caso al realizado por el lector consumado.

\section{Estudios instruccionales}

A través de dos estudios complementarios tratamos de introducir y evaluar experimentalmente un programa de instrucción en alumnos de BUP con diferente grado de habilidad lectora. El propósito principal de la primera investigación que desarrollamos fue comprobar si la adquisición de conocimiento específico sobre la estructura del texto, especialmente el relativo a las relaciones lógicas que gobiernan la información textual, sí como las estrategias que inciden en la detección de la macroestructura, presentes en los adultos expertos podrían ser enseñadas mediante una instrucción directa, explícita y sistemática en estos lectores. Se pretendía comprobar, asimismo, si ello revertiría en una mejora del producto lector tanto en los adolescentes competentes como en los menos capaces. Sobre este planteamiento diseñamos un programa de intervención dirigido a lectores adolescentes (1. ${ }^{\circ}$ BUP) y que denominamos Programa de Intervención Directa (ID). Más específicamente, con este programa se trata- ba de adiestrarles en habilidades que les permitiesen:

- Obtener un conocimiento específico sobre la estructura organizativa de algunos textos expositivos.

- Activar o generar el esquema general adecuado donde ubicar la información leída.

- Extraer las ideas principales del texto (la macroestructura).

- Identificar y retener las relaciones lógicas esenciales del contenido del pasaje y sus elementos principales.

- Ser capaz de elaborar un resumen adecuado, siguiendo la estructura original del texto.

Por otro lado, lo aplicamos dentro de un contexto social de interacción y bajo la instrucción razonada de habilidades propuesta por Brown y Palincsar (1982; Palincsar y Brown, 1984). Como complemento a estas dos perspectivas, este programa incluye, además, algunas tareas, como la titulación y el resumen, que han sido ampliamente utilizadas y difundidas, tanto en la instrucción de habilidades aisladas como en programas generales y completos.

Para llevar a cabo los objetivos que se pretendían alcanzar con la aplicación de este programa de instrucción, se incluían las siguientes tareas o actividades:

1: Elaboración del título del pasaje. La tarea del lector consistía en elaborar el título que mejor se adecuase a la información que se acababa de leer. Ello le exigía, en primer lugar, iniciar la búsqueda de un esquema general que, activado de abajo arriba (bottomup), tratase de recoger las características más importantes de la información leída.

2: Identificar la relación dominante del con. tenido del pasaje y sus elementos principales. Con esta tarea se introduce al alumno en el conocimiento y uso de la organización interna del texto, a través de algunas de las relaciones retóricas dominantes descritas por $\mathrm{Me}$ yer (1984, 1985a y b), características del material expositivo. Estas fueron: Respuesta: problema/solución. Causación: antecedente/consecuente. Colección y Descripción.

Una vez identificada la relación dominante y sus principales elementos, el lector de- 
bía extraer dicha relación. Para ello, el alumno se ayudaba de unos recuadros gráficos que, colocados en orden jerárquico según la estructura de cada texto de trabajo, debía situarla en su lugar correspondiente y completar, asimismo, la tarea, añadiendo en los diferentes recuadros los elementos principales y la información correspondiente a cada uno de ellos.

3: Elaborar un resumen del contenido leído. Con este último requisito se pretendía, además de asentar lo descrito hasta ahora, enseñar al adolescente a aplicar lo aprendido en la elaboración de un resumen. La tarea consistió en elaborar un extracto escrito del contenido del pasaje, siguiendo lo anotado en el esquema gráfico realizado. El lector, por tanto, utilizando tan sólo el título y la información esquemática en la que no debía considerar la información de detalle, debería realizar una síntesis por escrito que tratara de recoger exclusivamente lo más importante del texto. De esta manera, nos asegurábamos que el lector seguía el esquema organizativo del texto en la elaboración de sus respuestas.

Para la implantación de este programa en cada tarea se siguieron los pasos de la Instrucción Razonada de cada habilidad descrita por Brown y cols. (Brown y Palincsar, 1982; Palincsar y Brown, 1984) y constaba de tres fases consecutivas:

a.-Fase de explicación. El objetivo de esta fase no era otro que el de describir a los sujetos cada una de las tareas, su utilidad y finalidad de las mismas.

b.-Modelado. En esta fase se pretendía modular cada actividad en los alumnos. Primero a través de la ejecución del profesor para que, de manera progresiva, el alumno fuera internalizando la ejecución, asumiendo mayor responsabilidad en la tarea.

c.-Práctica supervisada. En esta fase se trataba de regular la aplicación en cada una de las tareas. El papel del investigador consistió en orientar al alumno en la realización de la tarea, señalando las cuestiones o errores que éste cometía, sugiriendo posibles estrategias, induciéndole a reflexionar y justificar sus decisiones, así como reforzar sus logros. A medida que estos lectores adolescentes conse- guían dominar la tarea, se les retiraban paulatinamente las ayudas prestadas por el experimentador.

Como aspectos complementarios a este propósito principal, deseábamos confirmar experimentalmente si la introducción de señalizaciones en el texto supone una ayuda a la intervención.

En este estudio participaron 72 alumnos de $1 .^{\circ}$ de BUP con dos niveles de comprensión ( 36 con buen nivel y 36 con un pobre nivel de comprensión) y se distribuyeron equitativamente en tres grupos: un grupo experimental y dos grupos control.

Los sujetos de cada nivel de competencia se asignaron de manera aleatoria a uno de los tres grupos que recibieron una de estas tres condiciones. Un primer grupo a la condición de «intervención directa» (ID). Un segundo grupo, asumió la condición de «intervención control» (IC). Este grupo, a diferencia del anterior, no se instruyó a los sujetos en ninguna estrategia o tarea encaminada a una mejora en el conocimiento en la estructura del texto o en una descripción razonada de habilidades. Muy al contrario, en esta intervención se reforzaba indiscriminadamente la estrategia utilizada por cada alumno, fuese cual fuese ésta. Por último, un tercer grupo asumió la condición de «sin intervención control» (SIC). Este grupo sólo recibió la aplicación de las V.V.D.D. Al igual que en otros estudios previos a todos los grupos se aplicaron dos pruebas de recuerdo libre: inmediato y demorado (7 días).

Los resultados obtenidos en este estudio se acomodan no sólo a la caracterización que habíamos establecido previamente entre los distintos niveles de competencia lectora, sino en la posible modificación de sus estrategias lectoras a través de este modelo de intervención. Así, los sujetos que recibieron el tratamiento ID incrementaron significativamente su recuerdo en las puntuaciones $\mathrm{R}$ sobre los dos grupos control $(p<0.01)$.

Esta mejora selectiva en las relaciones lógicas que gobiernan la estructura de los textos expositivos es en el único caso que se mantiene con independencia a la versión del pasaje aplicado o a la tarea de recuerdo asig- 


\section{4}

nada. Este hecho, sin embargo, recae fundamentalmente en los $\mathrm{BC}$, ya que en los sujetos $\mathrm{MC}$, aunque obtienen algunas mejoras respecto a sus compañeros de los grupos control, éstas no llegan a ser en todos los casos significativas.

Respecto a las señalizaciones, tanto los sujetos $\mathrm{BC}$ y MC de este tratamiento mejoran su recuerdo de la información relativa a estas mismas puntuaciones $\mathrm{R}$. Los lectores de este tratamiento obtienen también los mejores resultados en las puntuaciones $R$ en la tarea de recuerdo inmediata sobre los sujetos del grupo SIC, y en prácticamente en todas las medidas realizadas en la tarea con demora, siendo significativas sobre el tratamiento IC ( $p<0.001)$. Cuando a estos lectores se les prensenta la versión no señalizada, acusan en un primer momento la ausencia de tales ayudas, pero mejoran considerablemente en la segunda tarea de recuerdo. Esta es una características que sólo se observa en los sujetos $\mathrm{BC}$ de este tratamiento.

Sobre la organización del recuerdo, también son los lectores del tratamiento ID, tanto los $\mathrm{BC}$ como los $\mathrm{MC}$, los que más frecuentemente utilizan el plan organizacional del tex to en la elaboración de sus respuestas. Estas diferencias aumentan en la versión no señalizada y en el recuerdo con demora.

Este programa de intervención, por tanto, proporciona a los sujetos, especialmente a los que ya poseen una habilidad comprensiva aceptable (BC), una mejora considerable en la calidad del producto final lector, de manera similar a como lo realizaron los lectores más expertos en las puntuaciones $R$ sobre este mismo texto (véase León, 1991a). Este comportamiento se caracteriza por una mayor habilidad en la detección de la información relevante, mayor estabilidad del recuerdo con el paso del tiempo y en la frecuencia con la que siguen la estructura del pasaje cuando los lectores elaboran sus respuestas.

En un segundo estudio nos cuestionábamos la generalidad del programa de instrucción directa (ID). Nos preguntábamos cómo los diferentes grupos de sujetos que participaron en el estudio anterior se comportarían ante una situación nueva, ante la presentación de un texto organizado por una relación retórica (Comparación) no conocida, no explicada en ningún momento anterior. Por otro lado, algunos trabajos precedentes a éste han resaltado una diferencia importante entre el recuerdo de la información del texto y el aprendizaje del mismo (Kinstch, 1986; Mannes y Kintsch, 1987). En este sentido, un entrenamiento previo en estrategias lectoras y orientado hacia lo que va a ser estudiado inmediatamente después puede ayudar a los lectores a comprender y recordar mejor el material escrito. Sin embargo, si ese entrenamiento se organiza de manera diferente a la tarea que se le va a solicitar después, los resultados que se obtienen pueden ser aún mejores cuando se le exige al sujeto aplicar al lector lo aprendido ante situaciones nuevas.

Los resultados obtenidos reflejaron que los lectores que fueron entrenados mediante el modelo de Instrucción ID aplicaron sus conocimientos sobre la estructura del texto a otro cuya relación retórica dominante y sus principales elementos no habían sido previamente instruidas. Esta transferencia de lo aprendido a situaciones nuevas se manifiesta en un significativo incremento en el recuerdo de las ideas esenciales del texto y en los dos niveles de competencia sobre los grupos control; en un decremento de la información de detalle y en una mayor utilización por parte de estos lectores del plan organizativo del texto en la elaboración del recuerdo. El hecho de que el lector haya transferido los conocimientos adquiridos de manera relativamente diferente a como se les instruyera previamente ante situaciones nuevas, nos mueve a pensar que el lector consigue reconstruir una representación sobre el mundo o sobre la situación que se evoca en el texto (van Dijk y Kintsch, 1983).

En definitiva, los resultados de estos dos experimentos nos permiten defender las siguientes conclusiones:

1.-En general, los sujetos que se sometieron al programa de instrucción ID obtuvieron mejoras significativas en el recuerdo respecto a los grupos de control.

2.--Estas mejoras han sido selectivas en el sentido de que sólo afectan a aquellos aspec- 
tos que guardan relación con las estrategias instruidas, esto es, en una mayor y mejor retención de las principales relaciones lógicas y de las ideas centrales del texto, una mayor sensibilidad hacia las señalizaciones del texto y una aplicación más lógica y organizada en la elaboración de sus respuestas.

3.-Estas habilidades se generalizan a textos cuya estructura organizativa no había sido velada o instruida con anterioridad.

Todos estos cambios que observamos en los sujetos instruidos con este modelo de intervención corresponde a lo que podríamos deducir si se abandonara la estrategia de listado por la estrategia estructural: aplicar sus conocimientos sobre la organización interna del texto tanto en la detección, asimilación, recuperación y organización de las respuestas sobre la información leída como en la transferencia de esos conocimientos a situaciones nuevas. De esta forma, este nuevo comportamiento lector se aproxima al utilizado por los más expertos.

Este modelo de instrucción conlleva, sin embargo, algunas limitaciones. El modelo, aunque mejora significativamente el rendi- miento lector en los alumnos competentes, no mejora de manera significativa, en todos los casos, el rendimiento de los lectores menos capaces. Existen al menos dos aspectos que pueden haber influido en la eficacia del programa. El primero hace mención al número de sesiones administradas (cinco). Puede que este número de sesiones haya resultado insuficiente para permitir que los lectores menos competentes pudiesen modificar adecuadamente sus estrategias lectoras y aplicarlas ante la lectura de la nueva información. Un segundo factor hace referencia al número de sujetos que participaron en la intervención. Puede que en este caso el número resultase excesivo como para asegurar que la aplicación del programa ha sido llevada adecuadamente y por igual en todos los sujetos. En todo caso, estos problemas quedan abiertos a posibles investigaciones futuras que permitan esclarecer de una manera más precisa las causas de este hecho. Actualmente estamos llevando a cabo un nuevo programa que, impartido por varios profesores en sus respectivas asignaturas, trata de ofrecernos alguna información valiosa sobre los sujetos menos competentes.

\section{Notas}

${ }^{1}$ El lector interesado podrá encontrar información específica sobre las diferentes investigaciones en las notas a pie de página así como en la bibliografía que hemos sesgado intencionadamente hacia nuestros propios trabajos.

${ }^{2}$ En este apartado se expone una investigación actualmente en marcha en la que están colaborando Juan Ignacio Pozo, María del Puy Pérez Echeverría, Miguel Angel Gómez Crespo y Angeles Sanz. La realización de esta investigación es posible gracias a la financiación concedida por el C.I.D.E. al proyecto. «La comprensión de la química durante la adolescencia (12-16 años)», así como por la subvención n. ${ }^{\circ}$ PB90-0211 de la Dirección General de Investigación Científica y Técnica del M.E.C. a un proyecto supervisado por Juan Ignacio Pozo.

${ }^{3}$ Esta investigación corresponde a la Tesis Doctoral de J.A. León, dirigida por Mario Carretero.

\section{Referencias}

Anamuah-Mensah, J. (1986). «Cognitive strategies used by chemistry students to solve volumethric analysis problems." Journal of Research in Science Teaching, 23, (9), 759-769.

Archenhold, W.; Driver, R., y Orton, A. (Eds.) (1980). Cognitive development research in science and mathematics. Leeds: University of Leeds.

Asensio, M.; Carretero, M., y Pozo,J. I. (1989). «La comprensión del tiempo histórico». En M. Carretero, J. I. Pozo y M. Asensio (Comps.). La enseñanza de las Ciencias Sociales, Madrid: Visor.

Ausubel, D. P.; Novak, J. I. Y Hanesian, H. (1978). Educational Psychology. A cognitive view. Segunda edición. Nueva York: Holt, Rinehart y Winston (Psicología Educativa. Trad. cast. de M. Sandoval: México Trillas, 1983). 
BARTLETT, B. J. (1978) Top-level structure as an organizational strategy for recall of classroom text. Tesis doctoral no publicada. Arizona State University.

Bidell, T.R. y Fisher , K. W. (en prensa). «Cognitive development in contex: applying skill theory to education». En A. Demetriou, M. Shayer y A. Efklides (Eds.) The neopiagetian theories of cognitive development go to school. Londres: Routledge \& Kegan Paul.

Brooks, L. W. y Danseareau, D. F. (1983). "Effects of structural schema training and text organization on expository prose processing». Journal of Educational Psycbology, 75, (6), 811-820.

Brook , A; BRIGGS , B. (1983). Aspects of secondary students-understanding of the particulate nature of matter. Children's Learning in Science Proyet. Center for Studies in Science and Mathematics Education: The University of Leeds.

Brown, A. L.; DAY, J. D. y Jones, R.S. (1983). «The development of plans for summarizing text». Child Development, 54, 968-979.

Brown, S. L. y PALINCSAR, AS. (1982). «Inducing strategic learning from text by means of informed, selfcontrol training. Topics in Learning and Learming disabilities, 2,1-17.

CaREY, S (1985). Conceptual change in childbood. Cambridge, Mass.: The MIT Press.

Carretero, M. (1984). «De la larga distancia que separa la suposición de la certeza«. En: M. Carretero y J.A. García Madruga (Eds.). Lecturas de psicología del pensamiento. Madrid: Alianza.

Carretero, M. (1985). «El desarrollo cognitivo en la adolescencia y la juventud; las operaciones formales». En M. Carretero; A. Marchesi y J. Palacios (Eds.). Psicología Evolutiva 3. Adolescencia, Madurez, Senetud. Madrid: Alianza Psicología.

Carretero, M. (1989). «Procesamiento de la información y educación». Cuadernos de Pedalogía, 166, 78-83.

Carretero, M., Asensio, M. y Pozo, J. I. (1991). «Cognitive development, historical time representation and causal explanations in adolescence». En: M. Carretero, M. Pope, R. J. Simons y J. I. Pozo (Eds.). Leaming and Instruction. Vol.3. Pergamon Press.

Carretero, M. y Garcia Madrugada, J. A. (1984). Lecturas de Psicología del Pensamiento. Madrid: Alianza Universidad.

Carretero, M. y Leon, J. A. (1991). «Intervention in comprehension strategies: knowledge and use of the text structure.» Comunicación presentada al Fourth European Conference for Research on Learning and Instruction. Turku, Finlandia, 24-28 de agosto.

Carretero, M., Perez Echevarria, M. P. y Pozo, J. I. (1986). «El extraño caso del aceite de colza y la solución de problemas de correlación». Revista de Psicología General y Aplicada. 40, (4), 703-725.

Carretero, M.; Pozo, J. I. y Asensio, M. (1983). «Comprensión de conceptos históricos durante la adolescencia». Infancia y Aprendizaje, 23, 55-74.

CASE, R. (en prensa). "The role of central conceptual structures in the development of children's scientific and mathematical thought». En: A. Demetriou, M. Shayer y A. Efklides (Eds.). The cognitive development theories go to school. Londres Routledge \& Kegan Paul.

Demetriou, A.; Efrlides, A y Gustaffson, Ej. (en prensa). «Structural systems in developing cognition, science and education». En: A. Demetriuou, M. Shayer y A. Efklides (Eds.). The neopiagetian theories of cognitive development go to scbool. Londres: Rotuledge \& Kegan Paul.

van DiJK, T. A. y Kintsch, W. (1983). Strategies of discourse comprebension. Nueva York. Academic Press.

Driver, R. (1985). «Beyond Appearances: the conservation of matter under physical and chemical transformations». En Driver, E. Guesne y A. Tiberghien (Eds.) Children's ideas in science. Milton Keynes: Open University Press. Ideas cientificas en la infancia y la adolescencia. Madrid: Morata/MEC, 1989.

Driver, R., Guesne, E., y Tiberghien, A. (1985). Children's ideas in Science. Milton Keynes: Open University Press. (Trad. cast. de P. Manzano: Ideas científicas en la infancia y adolescencia. Madrid: Morata/MEC, 1989.

Engel Clough, E. y Driver, R. (1986). «A study of consistency in the use of students' conceptual frameworks across different task context.» Science Education, 70, (4), 473-496.

Flavell, J. H. (1982). «On cognitive development». Child Development, 53, 1-10.

Frazer, M. J. y SeRVANT, D. M. (1987). "Aspects of stoichiometry-where do students go wrong? Education in Chemistry, 24, (3), 73-75.

Furio, C y Hernandez, J.(1983). «Ideas sobre los gases en alumnos de 10 a 15 años.» Enseñanza de las Ciencias, $1,(2), 83-91$.

Furnham, A. F. (1988). Lay Theories. Everyday understanding of problems in the Social Sciences. Pergamon Press.

Garcia Madruga, J. A. y Carretero, M. (1986). «Estrategias en el razonamiento humano». En: H. Peraita (Coord.). Psicología Cognitiva y Ciencia Cognitiva. Madrid: U.N.E.D.

Garnham, A. (1987). Mental models as representation of discourse and text. Chichester: John Wiley \& Sons.

Geva, E. (1983). «Facilitating reading through flowcharting. Reading Research Quartely, 18, 384-405.

Hierrezuelo, J. y Montero, A. (1988). La ciencia de los alumnos. Barcelona: Laia/MEC.

Holland, J. M.; Holyoack, K. J.; Nisbett, R. E. y Thargard, P. R. (1986). Introduction Processes of inferen$c e$, learning and discovery. Cambridge, Mass.: The MIT Press.

Inhelder, B. y Piaget, J. (1955). De la logique e l'enfant a la logique de ladolescente. París: P.U.F. (Trad. cast. de M.T. Cevasco: De la lógica del niño a la lógica del adolescente. Buenos Aires: Paidós, 1972).

Jimenez AleixANDRE, M. P. (1990). Los esquemas conceptuales sobre la selección natural: análisis y propuestas para un cambio conceptual. Madrid: Tesis doctoral, facsímil, Universidad Complutense.

Johnson.Laird, P. N. (1983). Mental Models. Cambridge University Press. 
Just, M. A.y Carpenter, P. A. (1987). The Psychology of Reading and Language Comprebension. Massachusetts: Allyn and Bacon.

Karmiloff-Smith, A. e InHelder, B. (1974). «If you want to get ahead, get a theory». Cognition. 3, 195-212. (Trad. cast. de J. I. Pozo en Infancia y Aprendizaje. 1981, 13, 67-88).

KINTSCH, W. (1974). The representation of meaning in memory. Hillsdale, Nueva Jersey: Erebaum.

KINTSCH, W. (1986). «Learning from text». Cognition and Instruction, 3, 87-108.

KINTSCH, W. (1988). «The role of knowledge in discourse comprehension: a construction-integration model». Psychological Review, 95, (2), 163-182.

KINTSCH, W. y vaN DiJK, T. A. (1978). «Toward a model of text comprehension and production». Psychological Review, 85, 363-394.

Lawson, A. E. (1977). «Relationships among performances on three formal operational tasks». The Journal of Psychology, 96, 235-241.

LEON, J. A. (1989). Memoria y comprensión de textos: diferencias entre sujetos expertos y novatos. Tesis doctoral inédita. Universidad Autónoma de Madrid.

LEON, J. A. (1991a). «La comprensión del recuerdo de textos expositivos a través del análisis de algunas características del texto y del lector.» Infancia y Aprendizaje, 56, 51-76.

LEON, J. A. (1991b). «Intervención en estrategias de comprensión: un modelo basado en el conocimiento de la estructura del texto.» Infancia y Aprendizaje, 56, 77-91.

LEON, J. A. y CARRETERO, M. (en prensa) «Signals effects on the recall and understanding of expository text in expert and novice readersm. En: A. J. M. Oliviera (Ed.) Structures of comunication and intelligent belps for Hypermedia Couserware, Nueva York. Springer-Verlag.

LeOn, J. A. Lopez MAnjon, A. y CARRETERo, M. (1990). «El desarrollo intelectual durante la adolescencia: las operaciones formales». En: J.A. García Madruga y Pilar Lacasa (Comps.) Psicología Evolutiva. U.N.E.D.

LloRens, J.A. (1991). Comenzando a aprender química. De las ideas altemativas a las actividades de aprendizaje. Madrid: Visor.

LOMAN, N.C. y MAYER, R. (1983). «Signaling techniques that increase the understability of expository prose." Journal of Educational Psychology, 75, 402-412.

Lopez MANJON, A. (1991). Teorias intuitivas sobre la enfermedad: categorización, representación y razonamiento en expertos y novatos. Tesis doctoral. U.A.M.

Lopez Manjon, A. Carretero, M. (en prensa). «Teorías intuitivas sobre la gripe, el catarro y el SIDA: un estudio sobre expertos y profanos." Revista de Psicología Social.

MANDLER, J. M. y JoHNSON, N. S. (1977). «Remembrance of things parsed: story structure and recall». Cognitive Psychology, 9, 111-151.

ManNEs, S.S. y KINTSCH, W. (1987). «Knowledge organization and text organization». Cognition and Instruction, 4, (2), 91-115.

MAYER, R. E. (1985) «Structural analysis of science prose: can we increase problem-solving performance». En: B.K. Britton y J. B. Black (Eds.). Understanding expository text. Hillsdale, Nueva Yersey: Erlbaum.

MEYER, B. J. F. (1975). The organization of prose and its effects on memory. Amsterdam: Nort-Holland.

MEYER, B.J.F. (1984). «Text dimensions and cognitive processing». En: H. Mandl, N.L. Stein \& T. Trabasso (Eds.). Learning and comprehension of text. Hillsdale, N.J.; Erlbaum.

Meyer, B. J. F. (1985a) «Prose analysis: purposes, prodedures and problems. Part. I». En: B. K. Britton \& J. B. Black (Eds.) Understanding Expository Text. Hillsdale, Nueva Jersey: Erlbaum.

MEYER, B. J. F. (1975). «The organization of prose and its effects on memory. Ansterdam: North-Holland.

MEYeR, B. J. F. (1984)«Text dimensions and cognitive procesing». En: H. Mandl, N. L. Stein T. Trabasso (Eds.). Learning and comprebension of text. Hillsdale, N. J.: Erlbaum.

MeYer, B. J. F. (1985a). «Prose analysis: purposes, procedures and problems. Part I». En: B. K. Britton \& J. B. Black (Eds.) Undestanding Expository Text. Hillsdale, N. J.: Erlbaum.

MeYer, B. J. F. (1985b). «Prose analysis: purposes, procedures and problems. Part II». En: B. K. Britton \& J. B. Black (Eds.). Undestanding Expository Text. Hillsdale, Nueva Jersey: Erlbaum.

MeYer, B. J. F.; Young, C.J. y BARTLETT, B. J. (1989). Memory improved. Reading and memory enhancement across the life span through strategic text structures. Nueva Jersey: Erlbaum.

Oakhill, J. y Garnham, A. (1988), Becoming a skilled reader. Nueva York: Basil Blackwell.

PalinCSAR, A. S. y BROWN, A. L. (1984). «Reciprocal teaching of comprehension-fostering and comprehension-monitoring activities». Cognition and Instruction, 1, 117-175.

Perez Echeverria, M. P. (1990). Psicología del razonamiento probabilístico. Madrid: Servicio de Publicaciones de la Universidad Autónoma de Madrid.

PERRIG, W. y KINTSCH, W. (1985). «Propositional and situational representation of text.» Joumal of Memory and Language, 24, 503-518.

Pozo, J. I. (1987). Aprendizaje de la ciencia y Pensamiento Causal. Madrid: Aprendizaje Visor.

Pozo, J. I. (1988). «Procesos psicológicos en el cambios conceptual en química.» En Aspectos didácticos de la física y la química. Zaragoza: I. C. E. de la Universidad de Zaragoza.

Pozo, J. I., Asensio, M. y Carretero, M. (1989). La enseñanza de las Ciencias Sociales. Madrid: Visor.

Pozo, J. I. y Carretero, M. (1983). «El adolescente como historiador» Infancia y Aprendizaje, 23, 75-90.

Pozo, J. I. y CARretero, M. (1986). «El desarrollo intelectual durante la adolescencia». Cuadernos de Pedagogia, 133.

Pozo, J. I. y CARretero, M. (1987). «Del pensamiento formal a los concepciones espontáneas: ¿Qué cambia en la enseñanza de la ciencia?». Infancia y Aprendizaje, 38, 35-52. 
Pozo, J. I. y Carretero, M. (1992). «Causal theories, reasoning strategies and conflict resolution by experts and novices in newtonian mechanics». En: Demetriou, M. Shayer y A. Efklides (Eds.). Moderm theories of cognitive development go to school. London: Routledge y Kegan.

Pozo, J. I.; Gomez Crespo, M. A.; Limon, M. y SAnz, A. (1991a). Procesos cognitivos en la comprensión de la ciencia: ideas de los alumnos sobre la química. Madrid: Servicio de publicaciones del M. E. C.

Pozo, J. I., Perez Echeverria, M. P., Sanz, A. y Limon, M. (1992). «Las ideas de los alumnos sobre la ciencia como teorías implícitas.» Infancia y Aprendizaje, 57, 3-22.

Pozo, J.I.; Sanz, A., Gomez Crespo, M.A. Y Limon, M. (1991b). «Las ideas de los alumnos sobre la ciencia: una interpretación desde la psicología cognitiva». Enseñanza de las Ciencias, 9(1), 83-94.

Rosch, E. (1978) «Principles of categorization». En: E. Rosch y B. Lloyd (Eds.). Cognition and categorization. Hillsdale, N.J.: Erlbaum.

Rodrigo, M. J. (1985). «Las teorías implícitas en el conocimiento social». Infancia y Aprendizaje, 31-32, 145-156.

Rumelhart, D. E. (1975). «Notes on a schema for stories.» En: D. G. Bobrow y A. Collins (Eds.). Representation and Understanding Studies in cognitive Science, Nueva York: Academic Press.

SANZ, A. (1991). Razonamiento proporcional e influencia del contenido: un estudio en el campo de la quimica. Madrid: Universidad Autónoma, Memoria de Licenciatura inédita.

SCARDAMalia, M. y Bereiter, C. (1984). «Development of strategies in text processing.» En H. Mandl, N. L. Stein y T. Trabasso (Eds.). Learning and Comprebension of text. Hillsdale, Nueva Jersey: Erlbaum.

ScholNICK, E.K. (Ed.) (1983) New trends in conceptual representation: challenges to Piaget's theory? Hillsdale, N.J.: Erlbaum.

Sere, M. (1985). «The gaseous state». En: R. Driver, E. Guesne y A. Tiberghien (Eds.). Children's ideas in science. Milton Keynes: Open University Press. Trad. cast. de P. Manzano: Ideas cientificas en la infancia y la adolescencia. Madrid: Morata/MEC, 1989.

SERE, M. (1986). «Children's conceptions of the gaseous state, prior to teaching». European Journal of Science Education, 8, (4), 413-425.

Serrano, T. y BLANCO, A. (1988). Las ideas de los alumnos en el aprendizaje de las ciencias. Madrid: Narcea, apuntes IEPS, 47.

SHAYER, M. y ADEY, P. (1981). Towards a science of science teaching. Londres: Heinemann Educational Books.

SPRING, C. (1985). «Comprehension and study strategies reported by university freshmen who are good poor readers". Instructional Science, 14, 157-167.

Stein, N. L. y GLENN, C. G. (1979). «An analysis of story comprehension in elementary school children». En R. O. Freedle (Ed.) New directions in discourse Processing. Vol.2. Norwood. Nueva Jersey: Ablex.

TAYLOR, B. M. (1980). «Children's memory for expository tex after reading». Reading Research Quarterly, $15,399-441$.

TAYLOR, B. M. y BEACH, R. W. (1984). «The effects of text structure instruction on middle grade students comprehension and production of expository text». Reading Research Quarterly, 19, 134-146.

THORNDYKE, P.W. (1977) «Cognitive structures in comprehension and memory of narrative discourse». Cognitive Psychology, 9, 77-110.

TOURniaIRE, F. y PULOS, S. (1985). «Proportional reasoning: a review of the literature». Educational Studies in Mathematics, 16, 181-204.

Williams, J. P.; TAYLOR, M. B. y Ganger, S. (1981). «Text variations at the level of the individual sentence and the comprehension of simple expository paragraphs». Journal of Educational Psychology, 73, (6), 851-865.

\section{Extended Summary}

The aim of this paper is to introduce an approach to research on Psychology of Instruction being developed at the Autonoma University of Madrid. This line of research arose from studying some of the problems posed by Piagetian formal operations stage. The results of performance on formal operations tasks lead us to consider the importance of studying the content of logic tasks and of reasoning in general. This aspect also lead us to consider the study of specific contents in learning as an educational problem. A fundamental question asked was to what extent was the subjects' performance due to the use of general reasoning strategies over several contents or due to the subjects' causal theories about task content. To answer this question, two approaches were considered. First, research studies on expert and novice's performance in specific knowledge domains. Second, studies on students' alternative conceptions. These two approaches share a common concern for the content analysis of a problem allowing us a more detailed study of problem representation and problem solving. 
To illustrate the kind of work that has developed, two studies are presented. The first students studying Chemistry. Specifically, the objective is to analyze the pupils' ideas on three conceptual cores in Chemistry. These conceptual cores are: understanding the particular nature of matter, conservation of non-observable properties, and quantification of relationships. It is felt that they are related to most of the difficulties and conceptual mistakes found in these subjects. The second study is related to text comprehension and to several variables influencing it. This research study focuses more on general strategies than on the students' specific knowledge. It consists on an implementation and later an evaluation of an instructional programme aimed at improving text comprehension. The results showed an improvement in the trained comprehension strategies. Finally, it seems that our concerns have changed over time. These have developed from cognitive processes in reasoning to other aspects which take into account the importance of specific knowledge and therefore of instructional variables. 